\title{
ON THE DIMENSION OF GRAPHS OF WEIERSTRASS-TYPE FUNCTIONS WITH RAPIDLY GROWING FREQUENCIES
}

\author{
KRZYSZTOF BARAŃSKI
}

\begin{abstract}
We determine the Hausdorff and box dimension of the fractal graphs for a general class of Weierstrass-type functions of the form $f(x)=\sum_{n=1}^{\infty} a_{n} g\left(b_{n} x+\theta_{n}\right)$, where $g$ is a periodic Lipschitz real function and $a_{n+1} / a_{n} \rightarrow 0, b_{n+1} / b_{n} \rightarrow \infty$ as $n \rightarrow \infty$. Moreover, for any $H, B \in[1,2], H \leq B$ we provide examples of such functions with $\operatorname{dim}_{H}(\operatorname{graph} f)=\underline{\operatorname{dim}}_{B}(\operatorname{graph} f)=H, \overline{\operatorname{dim}}_{B}(\operatorname{graph} f)=B$.
\end{abstract}

\section{INTRODUCTION}

In this paper we study the dimension of the graphs of real functions of the form

$$
f: \mathbb{R} \rightarrow \mathbb{R}, \quad f(x)=\sum_{n=1}^{\infty} a_{n} g\left(b_{n} x+\theta_{n}\right),
$$

where $g: \mathbb{R} \rightarrow \mathbb{R}$ is a non-constant periodic Lipschitz function, $a_{n}, b_{n}>0$ with $b_{n+1} / b_{n} \rightarrow$ $\infty$ as $n \rightarrow \infty$ and $\theta_{n} \in \mathbb{R}$. The most well-known class of functions of the form (11) is the one with $b_{n}=b^{n}, a_{n}=b^{-\alpha n}$ for $b>1, \alpha \in(0,1)$, including the famous Weierstrass example of a continuous nowhere differentiable function on the interval. In spite of many efforts (see e.g. [4, 5, 6, 9, 10, and the references therein), the question of determining the Hausdorff dimension of the graphs of such functions is still (mostly) open.

It turns out that the case $b_{n+1} / b_{n} \rightarrow \infty$ is easier to handle. Probably the first to study such functions were Besicovitch and Ursell in 1937 [1], who considered the case when $g$ is the "sawtooth" function $\Lambda(x)=\operatorname{dist}(x, \mathbb{Z})$, with $a_{n}=b_{n}^{-\alpha}$ for some $\alpha \in(0,1)$ and $\theta_{n}=0$. In that case, they showed that if the sequence $b_{n+1} / b_{n}$ increases to $\infty$ and $\log b_{n+1} / \log b_{n} \rightarrow 1$ as $n \rightarrow \infty$, then the Hausdorff dimension of the graph of $f$ is equal to $2-\alpha$. Moreover, if $b_{n}=b_{1}^{\beta^{n-1}}$ where $b_{1}>1$ (then $\log b_{n+1} / \log b_{n} \rightarrow \beta$ as $\left.n \rightarrow \infty\right)$ and

$$
\beta=\frac{(1-\alpha)(2-H)}{\alpha(H-1)}
$$

for $1<H<2-\alpha$, then the graph of $f$ has Hausdorff dimension $H$.

In 1995, Wingren showed in [11] that the graph of the function

$$
f(x)=\sum_{n=1}^{\infty} 2^{-n} \Lambda\left(2^{2^{n}} x\right)
$$

Date: November 7, 2018.

2000 Mathematics Subject Classification. Primary 28A80, 28A78.

Research partially supported by Polish MNiSW Grant N N201 607940. 
has Hausdorff dimension equal to two (and so has any subset of the graph whose projection on the real axis has positive Lebesgue measure). In [7] Liu showed that any such subset of the graph of the function

$$
f(x)=\sum_{n=1}^{\infty} 2^{-n(n-1)-1} \Lambda\left(2^{n(n+1)+1} x\right)
$$

has Hausdorff dimension equal to one and infinite 1-Hausdorff measure.

Recently, Carvalho [2] showed that if $g$ is a periodic Lipschitz function, such that $g$ is increasing on some interval $I_{1}$ and decreasing on some interval $I_{2}$, with $|g(x)-g(y)|>$ $\delta|x-y|$ for every $x, y$ in $I_{1}$ and every $x, y$ in $I_{2}$, for some constant $\delta>0$, moreover $\log b_{n+1} / \log b_{n} \rightarrow \beta$ and $-\log b_{n} / \log a_{n} \rightarrow \alpha$ as $n \rightarrow \infty$ for $\alpha \in(0,1), \beta>1$, then

$$
\begin{aligned}
\operatorname{dim}_{H}(\operatorname{graph} f)= & \underline{\operatorname{dim}}_{B}(\operatorname{graph} f)=1+\frac{1-\alpha}{1-\alpha+\alpha \beta}, \\
& \overline{\operatorname{dim}}_{B}(\operatorname{graph} f)=2-\alpha,
\end{aligned}
$$

for the function $f$ of the form (1) (for arbitrary $\theta_{n}$ ). Here $\operatorname{dim}_{H}, \underline{\operatorname{dim}}_{B}$ and $\overline{\operatorname{dim}}_{B}$ denote respectively the Hausdorff, lower and upper box dimension (see Section 2 for definitions).

In this paper we complete the above results, determining the Hausdorff and box dimension of the graphs of functions of the form (1) in the general case $b_{n+1} / b_{n} \rightarrow \infty$, $a_{n+1} / a_{n} \rightarrow 0$. More precisely, we prove the following.

Theorem 1. Let $g: \mathbb{R} \rightarrow \mathbb{R}$ be a periodic Lipschitz function, such that $g$ is strictly monotone on some (non-trivial) interval $I \subset \mathbb{R}$ with $|g(x)-g(y)|>\delta|x-y|$ for every $x, y \in I$ and a constant $\delta>0$. If $a_{n}, b_{n}>0, a_{n+1} / a_{n} \rightarrow 0, b_{n+1} / b_{n} \rightarrow \infty$ as $n \rightarrow \infty$ and $\theta_{n} \in \mathbb{R}$, then for functions $f$ of the form (1) we have

$$
\begin{aligned}
\operatorname{dim}_{H}(\operatorname{graph} f)= & \underline{\operatorname{dim}}_{B}(\operatorname{graph} f)=1+\liminf _{n \rightarrow \infty} \frac{\log ^{+} d_{n}}{\log \left(b_{n+1} d_{n} / d_{n+1}\right)}, \\
& \overline{\operatorname{dim}}_{B}(\operatorname{graph} f)=1+\limsup _{n \rightarrow \infty} \frac{\log ^{+} d_{n}}{\log b_{n}},
\end{aligned}
$$

where

$$
d_{n}=a_{1} b_{1}+\cdots+a_{n} b_{n} .
$$

Remark 2. In fact, the proof shows that $\operatorname{dim}_{H}(\operatorname{graph} f)=\operatorname{dim}_{H}\left(\left.\operatorname{graph} f\right|_{\mathcal{I}}\right)$ for some Cantor set $\mathcal{I} \subset \mathbb{R}$ of Lebesgue measure 0 .

Remark 3. The assumptions on the function $g$ are satisfied, if $g$ is a periodic Lipschitz function, which is non-constant and $C^{1}$ on some interval (e.g. if $g$ is a periodic nonconstant $C^{1}$ function). Indeed, in this case there exists an interval $I$ with $\left.g^{\prime}\right|_{I}>\delta>0$ or $\left.g^{\prime}\right|_{I}<-\delta<0$.

Remark 4. The assertion on $\overline{\operatorname{dim}}_{B}\left(\right.$ graph $f$ ) holds under a weaker assumption: $a_{n+1} / a_{n} \rightarrow$ 0 can be replaced by $a_{n+1}<\eta a_{n}$ for large $n$, where $\eta$ is a sufficiently small constant depending on $g$ (not on the sequences $a_{n}, b_{n}, \theta_{n}$ ). 
Theorem 1 implies immediately the following corollaries.

Corollary 5. Under the assumption of Theorem 1, if additionally $a_{n+1} b_{n+1} \geq a_{n} b_{n} \geq 1$ for sufficiently large $n$, then

$$
\begin{aligned}
\operatorname{dim}_{H}(\operatorname{graph} f)= & \underline{\operatorname{dim}}_{B}(\operatorname{graph} f)=1+\liminf _{n \rightarrow \infty} \frac{\log \left(a_{n} b_{n}\right)}{\log \left(a_{n} b_{n} / a_{n+1}\right)}, \\
& \overline{\operatorname{dim}}_{B}(\operatorname{graph} f)=2-\limsup _{n \rightarrow \infty} \frac{\log a_{n}}{\log b_{n}},
\end{aligned}
$$

Proof. In this case $d_{n}>1$ and $a_{n} b_{n}<d_{n} \leq c n a_{n} b_{n}$ for some constant $c>0$, so

$$
0<\log ^{+} d_{n}-\log \left(a_{n} b_{n}\right)<\log n+\log c,
$$

which easily implies the assertion (see (4)).

Corollary 6. Under the assumption of Theorem 1, if additionally $\log b_{n+1} / \log b_{n} \rightarrow 1$ as $n \rightarrow \infty$, then

$$
\operatorname{dim}_{H}(\operatorname{graph} f)=\underline{\operatorname{dim}}_{B}(\operatorname{graph} f)=1+\liminf _{n \rightarrow \infty} \frac{\log ^{+} d_{n}}{\log b_{n}} .
$$

In this case $\operatorname{dim}_{B}(\operatorname{graph} f)$ exists if and only if there exists the limit $\gamma=\lim _{n \rightarrow \infty} \log ^{+} d_{n} / \log b_{n}$ and then

$$
\operatorname{dim}_{H}(\operatorname{graph} f)=\operatorname{dim}_{B}(\operatorname{graph} f)=1+\gamma .
$$

Proof. It is sufficient to show

$$
\lim _{n \rightarrow \infty} \frac{\log \left(b_{n+1} d_{n} / d_{n+1}\right)}{\log b_{n}}=1
$$

which is equivalent to

$$
\lim _{n \rightarrow \infty} \frac{\log \left(d_{n+1} / d_{n}\right)}{\log b_{n}}=0 .
$$

Since $a_{n+1} / a_{n} \rightarrow 0, b_{n+1} / b_{n} \rightarrow \infty$ as $n \rightarrow \infty$, we have $a_{n+1}<a_{n}$ and $b_{n+1}>b_{n}$ for large $n$, so

$0<\log \frac{d_{n+1}}{d_{n}}=\log \left(1+\frac{a_{n+1} b_{n+1}}{a_{1} b_{1}+\cdots+a_{n} b_{n}}\right)<\log \left(1+\frac{b_{n+1}}{b_{n}}\right)<\log b_{n+1}-\log b_{n}+\log 2$, which gives (2).

Corollary 7. Let $g$ be like in Theorem 1 and let

$$
f(x)=\sum_{n=1}^{\infty} b_{n}^{-\alpha} g\left(b_{n} x+\theta_{n}\right),
$$

where $\alpha \in(0,1], b_{n}>0, \theta_{n} \in \mathbb{R}$, and $b_{n+1} / b_{n} \rightarrow \infty$ as $n \rightarrow \infty$. Then

$$
\begin{aligned}
\operatorname{dim}_{H}(\operatorname{graph} f)= & \underline{\operatorname{dim}}_{B}(\operatorname{graph} f)=1+\frac{1-\alpha}{1-\alpha+\alpha \limsup _{n \rightarrow \infty} \log b_{n+1} / \log b_{n}}, \\
& \overline{\operatorname{dim}}_{B}(\operatorname{graph} f)=2-\alpha .
\end{aligned}
$$


(This includes the case $\lim \sup _{n \rightarrow \infty} \log b_{n+1} / \log b_{n}=\infty$ with the convention $1 / \infty=0$.) In particular, if $\log b_{n+1} / \log b_{n} \rightarrow 1$ as $n \rightarrow \infty$, then

$$
\operatorname{dim}_{H}(\operatorname{graph} f)=\operatorname{dim}_{B}(\operatorname{graph} f)=2-\alpha .
$$

Proof. Let $a_{n}=b_{n}^{-\alpha}$. Then $d_{n}=a_{1} b_{1}+\cdots+a_{n} b_{n}=b_{1}^{1-\alpha}+\cdots+b_{n}^{1-\alpha}$. Since $b_{n+1} / b_{n} \rightarrow \infty$, we have

$$
b_{n}^{1-\alpha}<d_{n}<2 b_{n}^{1-\alpha}
$$

for large $n$, which gives

$$
\lim _{n \rightarrow \infty} \frac{\log ^{+} d_{n}}{\log b_{n}}=1-\alpha
$$

and

$$
\begin{aligned}
\liminf _{n \rightarrow \infty} \frac{\log ^{+} d_{n}}{\log \left(b_{n+1} d_{n} / d_{n+1}\right)} & =\liminf _{n \rightarrow \infty} \frac{(1-\alpha) \log b_{n}}{\alpha \log b_{n+1}+(1-\alpha) \log b_{n}} \\
& =\frac{1-\alpha}{1-\alpha+\alpha \limsup _{n \rightarrow \infty} \log b_{n+1} / \log b_{n}}
\end{aligned}
$$

It is obvious that $\operatorname{dim}_{H}(\operatorname{graph} f), \overline{\operatorname{dim}}_{B}(\operatorname{graph} f) \in[1,2]$ and $\operatorname{dim}_{H}(\operatorname{graph} f) \leq \overline{\operatorname{dim}}_{B}(\operatorname{graph} f)$ (see Section 21). Using Theorem 1, we can provide examples of a function $f$ of the form (11) with any prescribed values of $\operatorname{dim}_{H}(\operatorname{graph} f)$ and $\overline{\operatorname{dim}}_{B}(\operatorname{graph} f)$.

Corollary 8. For every $H, B \in[1,2]$, such that $H \leq B$ there exists a function $f$ fulfilling the assumptions of Theorem 1, such that

$$
\operatorname{dim}_{H}(\operatorname{graph} f)=\underline{\operatorname{dim}}_{B}(\operatorname{graph} f)=H, \quad \overline{\operatorname{dim}}_{B}(\operatorname{graph} f)=B .
$$

Proof. A large part of examples is not new - we present them for completeness. The function $g$ can be taken to be e.g. sin, $\cos , \operatorname{dist}(\cdot, \mathbb{Z})$ etc.

For $H=B \in[1,2)$, it is enough to take

$$
f(x)=\sum_{n=1}^{\infty} n^{-(2-B) n} g\left(n^{n} x\right)
$$

and use Corollary 7 for $\alpha=2-B, b_{n}=n^{n}$. Similarly, for $H \in(1,2), B \in(H, 2)$ we take

$$
f(x)=\sum_{n=1}^{\infty} 2^{-(2-B) \beta^{n}} g\left(2^{\beta^{n}} x\right),
$$

where

$$
\beta=\frac{(2-H)(B-1)}{(H-1)(2-B)}
$$

and use Corollary 7 for $\alpha=2-B, b_{n}=2^{\beta^{n}}$. For $H=1, B \in(1,2)$ we take

$$
f(x)=\sum_{n=1}^{\infty} 2^{-(2-B) n^{n}} g\left(2^{n^{n}} x\right)
$$


and use Corollary 7 for $\alpha=2-B, b_{n}=2^{n^{n}}$.

For $H=1, B=2$, take

$$
f(x)=\sum_{n=1}^{\infty} 2^{-n^{n} / \sqrt{n}} g\left(2^{n^{n}} x\right),
$$

for $H \in(1,2), B=2$,

$$
f(x)=\sum_{n=1}^{\infty} 2^{-((2-H) /(e(H-1))) n^{n-1}} g\left(2^{n^{n}} x\right),
$$

and for $H=B=2$,

$$
f(x)=\sum_{n=1}^{\infty} n^{-\sqrt{n}} g\left(n^{n} x\right) .
$$

In all three cases, we have $a_{n+1} b_{n+1} /\left(a_{n} b_{n}\right) \rightarrow \infty$ as $n \rightarrow \infty$, so we can use Corollary 5 , Easy details are left to the reader.

The plan of the paper is as follows. After preliminaries, in Section 3, we prove some useful lemmas. The estimates for the box dimension are done in Section 4 (Propositions 19 201) and the Hausdorff dimension is estimated in Section 5 (Proposition 22 and Corollary 251). The proof follows the construction used by Mauldin and Williams in [9].

\section{Preliminaries}

For convenience, we recall the definitions of the Hausdorff and box dimension. For details, see e.g. [3, 8].

Definition 9. For $A \subset \mathbb{R}^{n}$ and $s>0$ the (outer) $s$-Hausdorff measure of $A$ is defined as

$$
\mathcal{H}^{s}(A)=\lim _{r \rightarrow 0^{+}} \inf \sum_{U \in \mathcal{U}}(\operatorname{diam} U)^{s},
$$

where infimum is taken over all countable coverings $\mathcal{U}$ of $A$ by open sets of diameters smaller than $r$. The Hausdorff dimension of $A$ is defined as

$$
\operatorname{dim}_{H}(A)=\sup \left\{s>0: \mathcal{H}^{s}(A)=+\infty\right\}=\inf \left\{s>0: \mathcal{H}^{s}(A)=0\right\} .
$$

Definition 10. For a bounded set $A \subset \mathbb{R}^{n}$ and $r>0$ let $N(r)$ be the minimal number of balls of diameter $r$ needed to cover $A$. The lower and upper box dimension (also called the box-counting or Minkowski dimension) are defined respectively as

$$
\underline{\operatorname{dim}}_{B}(A)=\liminf _{r \rightarrow 0^{+}} \frac{\log N(r)}{-\log r}, \quad \overline{\operatorname{dim}}_{B}(A)=\limsup _{r \rightarrow 0^{+}} \frac{\log N(r)}{-\log r} .
$$

The lower and upper box dimension dimension of an arbitrary set $A \subset \mathbb{R}^{n}$ are defined as

$$
\underline{\operatorname{dim}}_{B}(A)=\sup _{X \text { bounded }} \underline{\operatorname{dim}}_{B}(A \cap X), \quad \overline{\operatorname{dim}}_{B}(A)=\sup _{X \text { bounded }} \overline{\operatorname{dim}}_{B}(A \cap X) .
$$

If $\underline{\operatorname{dim}}_{B}(A)=\overline{\operatorname{dim}}_{B}(A)$, then the common value is called the box dimension of $A$ and is denoted by $\operatorname{dim}_{B}(A)$. 
We have

$$
\operatorname{dim}_{H}(A) \leq \underline{\operatorname{dim}}_{B}(A) .
$$

The definitions of the Hausdorff and box dimension easily imply

Lemma 11. Let $f:[a, b] \rightarrow \mathbb{R}$ for $a, b \in \mathbb{R}, a<b$. Then $\operatorname{dim}_{H}(\operatorname{graph} f) \geq 1$. Moreover, if $f$ is is Hölder continuous with exponent $\alpha \in(0,1]$, i.e.

$$
\|f(x)-f(y)\| \leq c\|x-y\|^{\alpha}
$$

for every $x, y \in A$ and some constant $c>0$, then

$$
\overline{\operatorname{dim}}_{B}(\operatorname{graph} f) \leq 2-\alpha .
$$

In particular, if $\alpha=1$, i.e. $f$ is Lipschitz continuous, then

$$
\operatorname{dim}_{H}(\operatorname{graph} f)=\operatorname{dim}_{B}(\operatorname{graph} f)=1 .
$$

Definition 12. The Hausdorff dimension of a finite (non-zero) Borel measure $\nu$ in $\mathbb{R}^{n}$ is defined as

$$
\operatorname{dim}_{H}(\nu)=\inf \left\{\operatorname{dim}_{H}(A): A \text { has full measure } \mu\right\} .
$$

A well-known tool for estimating the Hausdorff dimension is the following fact (see e.g. [3, 8]).

Lemma 13. Let $B_{r}(x)$ denote the ball in $\mathbb{R}^{n}$ centred at $x$ of radius $r$. If

$$
\liminf _{r \rightarrow 0^{+}} \frac{\log \nu\left(B_{r}(x)\right)}{\log r} \geq D
$$

for $\nu$-almost every $x$, then $\operatorname{dim}_{H}(\nu) \geq D$, in particular $\operatorname{dim}_{H}(A) \geq D$ for every Borel set A of positive measure $\nu$. The ball $B_{r}(x)$ can be replaced by an $n$-dimensional cube centred at $x$ of side $r$.

Notation. We set $\mathbb{N}=\{1,2,3, \ldots\}$. By card we denote the cardinality of a set. The integer part of $x \in \mathbb{R}$ (i.e. the largest integer not larger than $x$ ) is denoted by $[x]$. The 1-dimensional Lebesgue measure of a set $A \subset \mathbb{R}$ is denoted by $|A|$.

\section{LEMMAS}

Let $f$ satisfy the assumptions of Theorem 11. Obviously, we can assume that the period of $g$ is equal to 1 , the interval $I$ is closed and contained in $[0,1]$ and $g$ is strictly increasing on $I$. Since $g$ is Lipschitz and

$$
\operatorname{dim}_{H}(\operatorname{graph}(f+h))=\operatorname{dim}_{H}(\operatorname{graph} f), \quad \operatorname{dim}_{B}(\operatorname{graph}(f+h))=\operatorname{dim}_{B}(\operatorname{graph} f)
$$

for every Lipschitz function $h: \mathbb{R} \rightarrow \mathbb{R}$ (see e.g. [9]), taking $h=\sum_{n=1}^{N} a_{n} g\left(b_{n} x+\theta_{n}\right.$ ) for any $N \geq 1$, we can replace $f$ by $\sum_{n=N+1}^{\infty} a_{n} g\left(b_{n} x+\theta_{n}\right)$. Therefore, we can assume

$$
\frac{a_{n+1}}{a_{n}}<\eta, \quad \frac{b_{n+1}}{b_{n}}>\frac{1}{\eta}
$$


for an arbitrarily small fixed $\eta>0$ (cf. Remark 4). Note also that by the Stolz-Cesáro theorem,

$$
\lim _{n \rightarrow \infty} \frac{n}{\log b_{n}}=\lim _{n \rightarrow \infty} \log \frac{b_{n}}{b_{n+1}}=0 .
$$

Lemma 14. We have

$$
\frac{d_{n+1}}{b_{n+1}}<2 \eta \frac{d_{n}}{b_{n}}
$$

for $\eta$ from (3). In particular, $d_{n} / b_{n} \rightarrow 0$ as $n \rightarrow \infty$.

Proof. By (3), we have

$$
\frac{d_{n+1} b_{n}}{d_{n} b_{n+1}}=\left(1+\frac{a_{n+1} b_{n+1}}{a_{1} b_{1}+\cdots+a_{n} b_{n}}\right) \frac{b_{n}}{b_{n+1}}<\frac{b_{n}}{b_{n+1}}+\frac{a_{n+1}}{a_{n}}<2 \eta<1,
$$

if $\eta$ is chosen sufficiently small.

Lemma 15. There exists a constant $c_{0}>0$, such that for every $x, y \in \mathbb{R}$ and every $n \in \mathbb{N}$,

$$
|f(x)-f(y)| \leq c_{0}\left(d_{n}|x-y|+a_{n+1}\right) .
$$

Proof. Let $f_{i}(t)=a_{i} g\left(b_{i} t+\theta_{i}\right)$ for $i \in \mathbb{N}$. Then

$$
\left|f_{i}(x)-f_{i}(y)\right| \leq L a_{i} b_{i}|x-y|
$$

where $L$ is the Lipschitz constant of $g$. This together with (3) implies

$$
\begin{aligned}
& |f(x)-f(y)| \leq \sum_{i=1}^{n}\left|f_{i}(x)-f_{i}(y)\right|+2 \sup \left|f-\sum_{i=1}^{n} f_{i}\right| \\
& \quad \leq L d_{n}|x-y|+2 \sup |g| \sum_{i=n+1}^{\infty} a_{i}<L d_{n}|x-y|+\frac{2 \sup |g|}{1-\eta} a_{n+1}<c_{0}\left(d_{n}|x-y|+a_{n+1}\right)
\end{aligned}
$$

for a suitable constant $c_{0}>0$.

Let

$$
I_{0,0}=I, \quad I_{n, j}=\frac{I-\theta_{n}+j}{b_{n}}
$$

for $n \in \mathbb{N}, j \in \mathcal{J}_{n}$, where $\mathcal{J}_{n} \subset \mathbb{Z}$ is defined inductively as

$$
\mathcal{J}_{0}=\{0\}, \quad \mathcal{J}_{n}=\left\{j \in \mathbb{Z}: I_{n, j} \subset I_{n-1, j^{\prime}} \text { for some } j^{\prime} \in \mathcal{J}_{n-1}\right\}
$$

for $n \in \mathbb{N}$. Then every $I_{n, j}$ is an interval of length $|I| / b_{n}$ and the gap between two consecutive intervals $I_{n, j}, I_{n, j+1} \subset I_{n-1, j^{\prime}}$ has length $(1-|I|) / b_{n}$. By definition,

$$
b_{n} x+\theta_{n} \in I \quad \bmod 1 \quad \text { for every } x \in I_{n, j} .
$$

We will call $I_{n, j}$ intervals of $n$-th generation. By (3), we can assume that every interval of $n$-th generation contains at least two intervals of next generation. 
Lemma 16. There exists $q>0$ such that the interval $I$ contains more than $q b_{1}$ intervals of first generation and for every $n \in \mathbb{N}$, every interval of $n$-th generation contains more than $q b_{n+1} / b_{n}$ intervals of $(n+1)$-th generation. Moreover,

$$
\text { card } \mathcal{J}_{n}>q^{n} b_{n}
$$

Proof. For $n \geq 0, j \in \mathcal{J}_{n}$ let $N_{n, j}$ be the number of intervals of $(n+1)$-th generation contained in $I_{n, j}$. Since the intervals of $(n+1)$-th generation have length $|I| / b_{n+1}$ and are separated by gaps of length at least $(1-|I|) / b_{n+1}$, we have, setting $b_{0}=1$,

$$
\frac{|I|}{b_{n}}<\frac{N_{n, j}+2}{b_{n+1}}
$$

so by (3),

$$
N_{n, j}>|I| \frac{b_{n+1}}{b_{n}}-2>q \frac{b_{n+1}}{b_{n}}
$$

for some constant $q>0$. Moreover,

$$
\operatorname{card} \mathcal{J}_{n} \geq N_{0,0} \min _{j_{1} \in \mathcal{J}_{1}} N_{1, j_{1}} \cdots \min _{j_{n-1} \in \mathcal{J}_{n-1}} N_{n-1, j_{n-1}}>q^{n} b_{n} .
$$

Lemma 17. There exist $c_{1}, c_{2}>0$, such that for every $n \in \mathbb{N}, j \in \mathcal{J}_{n}$ and every $x, y \in I_{n, j}$,

$$
|f(x)-f(y)| \geq c_{1} d_{n}|x-y|-c_{2} a_{n+1} .
$$

Proof. We can assume $x>y$. Since every interval of $n$-th generation is contained in an interval of $i$-th generation for every $i \leq n$, by (5) we have

$$
f_{i}(x)-f_{i}(y)>\delta a_{i} b_{i}(x-y) \quad \text { for } i=1, \ldots, n,
$$

SO

$$
\begin{aligned}
f(x)-f(y)>\delta d_{n}(x-y)-2 \sup & \left|f-\sum_{i=1}^{n} f_{i}\right| \geq \delta d_{n}(x-y)-2 \sup |g| \sum_{i=n+1}^{\infty} a_{i} \\
& >\delta d_{n}(x-y)-\frac{2 \sup |g|}{1-\eta} a_{n+1}>c_{1} d_{n}|x-y|-c_{2} a_{n+1}
\end{aligned}
$$

for suitable constants $c_{1}, c_{2}>0$.

\section{Box DIMENSION}

Now we prove (simultaneously) two following propositions.

Proposition 18. Let $\bar{\gamma}=\lim \sup _{n \rightarrow \infty} \log ^{+} d_{n} / \log b_{n}$. Then the following assertions hold:

(a) The function $f$ is Hölder continuous with every exponent smaller than $1-\bar{\gamma}$ and is not Hölder continuous with any exponent larger than $1-\bar{\gamma}$.

(b) If $a_{n}=b_{n}^{-\alpha}$ for some $\alpha \in(0,1]$, then $f$ is Hölder continuous with exponent $\alpha=1-\bar{\gamma}$.

(c) If the sequence $d_{n}$ is bounded, then $f$ is Lipschitz continuous, so $\operatorname{dim}_{H}(\operatorname{graph} f)=$ $\operatorname{dim}_{B}(\operatorname{graph} f)=1$. 
Proposition 19. We have

$$
\overline{\operatorname{dim}}_{B}(\operatorname{graph} f)=1+\limsup _{n \rightarrow \infty} \frac{\log ^{+} d_{n}}{\log b_{n}} .
$$

Proof. For a set $A \subset \mathbb{R}$ let

$$
V_{A}=\sup _{A} f-\inf _{A} f
$$

Take a small $r>0$. Let $k=k(r) \in \mathbb{N}$ be such that

$$
\frac{1}{b_{k+1}} \leq r<\frac{1}{b_{k}}
$$

By Lemma 15, for every $t \in \mathbb{R}$ we have

$$
V_{[t, t+r]} \leq c_{0}\left(d_{k} r+a_{k+1}\right)<c_{0}\left(d_{k} r+\frac{d_{k+1}}{b_{k+1}}\right) \leq 2 c_{0} \max \left(d_{k} r, \frac{d_{k+1}}{b_{k+1}}\right) .
$$

In particular, if the sequence $d_{n}$ is bounded, then (66) and (7) give

$$
V_{[t, t+r]}<2 c_{0} \max \left(d r, \frac{d}{b_{k+1}}\right) \leq 2 c_{0} d r
$$

for some constant $d>0$, which means that $f$ is Lipschitz and proves the assertion (c) of Proposition 18. Hence, we assume from now on that $d_{n} \rightarrow \infty$ as $n \rightarrow \infty$, in particular $d_{n}>1$ and $\log ^{+} d_{n}=\log d_{n}$ for large $n$.

By (6) and Lemma 14, we have

$$
\begin{aligned}
d_{k} r & =r^{1+\log d_{k} / \log r}<r^{1-\log d_{k} / \log b_{k}}, \\
\frac{d_{k+1}}{b_{k+1}} & =\left(\frac{1}{b_{k+1}}\right)^{1-\log d_{k+1} / \log b_{k+1}} \leq r^{1-\log d_{k+1} / \log b_{k+1}} .
\end{aligned}
$$

Hence, (77) implies

$$
V_{[t, t+r]} \leq 2 c_{0} r^{1-\max \left(\log d_{k} / \log b_{k}, \log d_{k+1} / \log b_{k+1}\right)} .
$$

On the other hand, by Lemma 17, (3) and Lemma 14, for every $n \in \mathbb{N}, j \in \mathcal{J}_{n}$ we have

(9) $\quad V_{I_{n, j}} \geq c_{1}|I| \frac{d_{n}}{b_{n}}-c_{2} a_{n+1}>c_{1}|I| \frac{d_{n}}{b_{n}}-c_{2} \eta a_{n}>\left(c_{1}|I|-\eta c_{2}\right) \frac{d_{n}}{b_{n}}$

$$
=\left(c_{1}|I|-\eta c_{2}\right)\left(\frac{1}{b_{n}}\right)^{1-\log d_{n} / \log b_{n}}>\left(c_{1}|I|-\eta c_{2}\right)\left|I_{n, j}\right|^{1-\log d_{n} / \log b_{n}},
$$

where $c_{1}|I|-\eta c_{2}>0$, if $\eta$ was chosen sufficiently small.

Let $s<1-\bar{\gamma}$. Then for sufficiently large $k$ we have

$$
1-\max \left(\log d_{k} / \log b_{k}, \log d_{k+1} / \log b_{k+1}\right)>s,
$$

so by (8), the function $f$ is Hölder continuous with exponent $s$.

Take now $s>1-\bar{\gamma}$ and suppose $f$ is Hölder continuous with exponent $s$. Then there exists $\varepsilon>0$ such that $1-\log d_{n} / \log b_{n}<s-\varepsilon$ for infinitely many $n$, which contradicts (9). 
In this way we have proved the assertion (a) of Proposition 18, Note that the assertion (a) implies immediately that

$$
\overline{\operatorname{dim}}_{B}(\operatorname{graph} f) \leq 1+\bar{\gamma}
$$

(see Lemma 11). If $a_{n}=b_{n}^{-\alpha}$ for some $\alpha \in(0,1]$, then $d_{n}=b_{1}^{1-\alpha}+\cdots+b_{n}^{1-\alpha}$, so (since $\left.b_{n+1} / b_{n} \rightarrow \infty\right)$, we have

$$
b_{n}^{1-\alpha}<d_{n}<2 b_{n}^{1-\alpha}
$$

for large $n$. Hence, $\alpha=1-\bar{\gamma}$ and, by (6) and (7),

$$
V_{[t, t+r]}<4 c_{0} \max \left(b_{k}^{1-\alpha} r, b_{k+1}^{-\alpha}\right) \leq 4 c_{0} r^{\alpha},
$$

which proves the assertion (b) of Proposition 18.

Let $N(r)$ be the minimal number of squares with vertical and horizontal sides of length $r$ needed to cover graph $\left.f\right|_{I}$. Since, by Lemma 16, for every $n$ there are more than $q^{n} b_{n}$ disjoint intervals $I_{n, j}$ of lengths $|I| / b_{n}$ contained in $|I|$, using (9) we get

$$
N\left(\left|I_{n, j}\right|\right)>c q^{n} b_{n}\left|I_{n, j}\right|^{-\log d_{n} / \log b_{n}}
$$

for some constant $c>0$, so (using (4)) we have

$$
\begin{aligned}
\overline{\operatorname{dim}}_{B}(\operatorname{graph} f) \geq \limsup _{n \rightarrow \infty} & \frac{\log N\left(\left|I_{n, j}\right|\right)}{-\log \left|I_{n, j}\right|} \\
& \geq \limsup _{n \rightarrow \infty} \frac{n \log q+\log b_{n}+\log d_{n}\left(1-\log |I| / \log b_{n}\right)}{\log b_{n}}=1+\bar{\gamma} .
\end{aligned}
$$

This together with (10) gives

$$
\overline{\operatorname{dim}}_{B}(\operatorname{graph} f)=1+\bar{\gamma}
$$

which proves Proposition 19.

Proposition 20. We have

$$
\underline{\operatorname{dim}}_{B}(\operatorname{graph} f) \leq 1+\liminf _{n \rightarrow \infty} \frac{\log ^{+} d_{n}}{\log \left(b_{n+1} d_{n} / d_{n+1}\right)} .
$$

Proof. Take a small $r>0$. Let $k=k(r), m=m(r) \in \mathbb{N}$ be such that

$$
\frac{1}{b_{k+1}} \leq r<\frac{1}{b_{k}}, \quad \frac{m}{b_{k+1}} \leq r<\frac{m+1}{b_{k+1}} .
$$

Obviously, $k \rightarrow \infty$ as $r \rightarrow 0^{+}$. By definition, $m \in\left\{1, \ldots, m_{k}\right\}$, where

$$
m_{k}= \begin{cases}b_{k+1} / b_{k}-1 & \text { if } b_{k+1} / b_{k} \in \mathbb{N} \\ {\left[b_{k+1} / b_{k}\right]} & \text { otherwise. }\end{cases}
$$

By Proposition 18 (c), we can assume that $d_{k}>1$. By (17), for every $t \in \mathbb{R}$

$$
V_{[t, t+r]}<2 c_{0} \max \left(\frac{(m+1) d_{k}}{b_{k+1}}, \frac{d_{k+1}}{b_{k+1}}\right) \leq 4 c_{0} \max \left(\frac{m d_{k}}{b_{k+1}}, \frac{d_{k+1}}{b_{k+1}}\right),
$$


so by (11),

$$
\begin{aligned}
\underline{\operatorname{dim}}_{B}(\operatorname{graph} f) & \leq 2+\liminf _{r \rightarrow 0^{+}} \sup _{t \in \mathbb{R}} \frac{\log V_{[t, t+r]}}{-\log r} \\
& \leq 2+\liminf _{r \rightarrow 0^{+}} \max \left(\frac{\log \left(m d_{k} / b_{k+1}\right)}{\log \left(b_{k+1} / m\right)}, \frac{\log \left(d_{k+1} / b_{k+1}\right)}{\log \left(b_{k+1} / m\right)}\right) \\
& =1+\liminf _{r \rightarrow 0^{+}} \max \left(\frac{\log d_{k}}{\log \left(b_{k+1} / m\right)}, \frac{\log \left(d_{k+1} / m\right)}{\log \left(b_{k+1} / m\right)}\right) .
\end{aligned}
$$

To end the proof of the proposition, it remains to use Lemma 21, which we prove below.

Lemma 21. For $r>0$ and $k=k(r), m=m(r)$ defined in (11), we have

$$
\liminf _{r \rightarrow 0^{+}} \max \left(\frac{\log d_{k}}{\log \left(b_{k+1} / m\right)}, \frac{\log \left(d_{k+1} / m\right)}{\log \left(b_{k+1} / m\right)}\right)=\liminf _{n \rightarrow \infty} \frac{\log d_{n}}{\log \left(b_{n+1} d_{n} / d_{n+1}\right)} .
$$

Proof. For $r$ such that $k=k(r)$ is constant, (i.e. $r \in\left[1 / b_{k+1}, 1 / b_{k}\right)$ ), let

$$
X_{k}(m)=X_{k}(m(r))=\frac{\log d_{k}}{\log \left(b_{k+1} / m\right)}, \quad Y_{k}(m)=Y_{k}(m(r))=\frac{\log \left(d_{k+1} / m\right)}{\log \left(b_{k+1} / m\right)}
$$

for $m=m(r) \in\left\{1, \ldots, m_{k}\right\}$ and let

$$
M_{k}=\min _{m \in\left\{1, \ldots, m_{k}\right\}} \max \left(X_{k}(m), Y_{k}(m)\right) .
$$

It is clear that

$$
\liminf _{r \rightarrow 0^{+}} \max \left(X_{k}(m), Y_{k}(m)\right)=\liminf _{r \rightarrow 0^{+}} M_{k} .
$$

Note that $X_{k}$ is an increasing function of $m$, while $Y_{k}$ is a decreasing one (since, by Lemma 14, we have $\left.d_{k+1}<b_{k+1}\right)$. Moreover,

$$
X_{k}(1)=\frac{\log d_{k}}{\log b_{k+1}}<\frac{\log d_{k+1}}{\log b_{k+1}}=Y_{k}(1)
$$

and

$$
X_{k}(m) \leq Y_{k}(m) \Longleftrightarrow m \leq \frac{d_{k+1}}{d_{k}} .
$$

By Lemma 14 and (3), we have

SO

$$
1<\frac{d_{k+1}}{d_{k}}<2 \eta \frac{b_{k+1}}{b_{k}}<\frac{b_{k+1}}{b_{k}}-2<m_{k},
$$

$$
\left[\frac{d_{k+1}}{d_{k}}\right] \geq 1, \quad\left[\frac{d_{k+1}}{d_{k}}\right]+1 \leq m_{k}
$$

Hence,

$$
M_{k}=\min \left(Y_{k}\left(\left[\frac{d_{k+1}}{d_{k}}\right]\right), X_{k}\left(\left[\frac{d_{k+1}}{d_{k}}\right]+1\right)\right) .
$$


Consider the condition

$$
d_{k+1} \geq 2 d_{k}
$$

If (14) is not satisfied, then $\left[d_{k+1} / d_{k}\right]=1$ and (13) gives

$$
M_{k}=\min \left(Y_{k}(1), X_{k}(2)\right)=\min \left(\frac{\log d_{k+1}}{\log b_{k+1}}, \frac{\log d_{k}}{\log \left(b_{k+1} / 2\right)}\right)=\frac{\log d_{k+1}}{\log b_{k+1}}+o(1)
$$

as $r \rightarrow 0^{+}$. If (14) is satisfied, then

$$
\frac{d_{k+1}}{2 d_{k}}<\left[\frac{d_{k+1}}{d_{k}}\right]<\left[\frac{d_{k+1}}{d_{k}}\right]+1<\frac{2 d_{k+1}}{d_{k}}
$$

SO

$$
\frac{\log d_{k}}{\log \left(b_{k+1} d_{k} / d_{k+1}\right)} \leq Y_{k}\left(\left[\frac{d_{k+1}}{d_{k}}\right]\right)<\frac{\log \left(2 d_{k}\right)}{\log \left(2 b_{k+1} d_{k} / d_{k+1}\right)}
$$

and

$$
\frac{\log d_{k}}{\log \left(b_{k+1} d_{k} / d_{k+1}\right)}<X_{k}\left(\left[\frac{d_{k+1}}{d_{k}}\right]+1\right)<\frac{\log d_{k}}{\log \left(b_{k+1} d_{k} /\left(2 d_{k+1}\right)\right)} .
$$

Suppose first that (14) does not hold for almost all $k$. Then for all $k$ we have $\log d_{k} \leq$ $k \log 2+c$ for some constant $c>0$, so by (44), both sides of the equation in the lemma are equal to 0 . Hence, we can assume that (14) holds for infinitely many $k$. If it holds for almost all $k$, then (13), (16) and (17) give

$$
\liminf _{r \rightarrow 0^{+}} M_{k}=\liminf _{r \rightarrow 0^{+}} \frac{\log d_{k}}{\log \left(b_{k+1} d_{k} / d_{k+1}\right)}=\liminf _{n \rightarrow \infty} \frac{\log d_{n}}{\log \left(b_{n+1} d_{n} / d_{n+1}\right)},
$$

which (together with (12)) ends the proof in this case. Otherwise, (14) does not hold for infinitely many $k$, and then (13), (15), (16) and (17) give

$$
\liminf _{r \rightarrow 0^{+}} M_{k}=\min \left(\liminf _{r \rightarrow 0^{+}} \frac{\log d_{k}}{\log b_{k}}, \liminf _{r \rightarrow 0^{+}} \frac{\log d_{k}}{\log \left(b_{k+1} d_{k} / d_{k+1}\right)}\right) .
$$

By Lemma 14,

SO

$$
\frac{\log d_{k}}{\log b_{k}}>\frac{\log d_{k}}{\log \left(b_{k+1} d_{k} / d_{k+1}\right)}
$$

$$
\liminf _{r \rightarrow 0^{+}} \frac{\log d_{k}}{\log b_{k}} \geq \liminf _{r \rightarrow 0^{+}} \frac{\log d_{k}}{\log \left(b_{k+1} d_{k} / d_{k+1}\right)}
$$

Hence,

$$
\liminf _{r \rightarrow 0^{+}} M_{k}=\liminf _{r \rightarrow 0^{+}} \frac{\log d_{k}}{\log \left(b_{k+1} d_{k} / d_{k+1}\right)}=\liminf _{n \rightarrow \infty} \frac{\log d_{n}}{\log \left(b_{n+1} d_{n} / d_{n+1}\right)},
$$

which (together with (12)) ends the proof.

Note that in this section, instead of the assumption $a_{n+1} / a_{n} \rightarrow 0$, we used a weaker condition (3). This proves Remark 4 . 


\section{HAusdorfF Dimension}

Let $\mathcal{I}$ be the Cantor set defined as

$$
\mathcal{I}=\bigcap_{n=0}^{\infty} \bigcup_{j \in \mathcal{J}_{n}} I_{n, j} .
$$

It is obvious by construction that $\mathcal{I}$ has Lebesgue measure 0 (cf. Remark 21). Let $\mu$ be the probabilistic Borel measure in $\mathbb{R}$ supported on $\mathcal{I}$, such that for every $I_{n+1, j^{\prime}} \subset I_{n, j}$ with $j \in \mathcal{J}_{n}, j^{\prime} \in \mathcal{J}_{n+1}$, we have

$$
\mu\left(I_{n+1, j^{\prime}}\right)=\frac{\mu\left(I_{n, j}\right)}{\operatorname{card}\left\{j^{\prime \prime} \in \mathcal{J}_{n+1}: I_{n+1, j^{\prime \prime}} \subset I_{n, j}\right\}}
$$

(the construction of such a measure is standard). By Lemma 16, we have

$$
\mu\left(I_{n, j}\right)<\frac{1}{q^{n} b_{n}}
$$

for every $n \in \mathbb{N}, j \in \mathcal{J}_{n}$. Let $\nu$ be a probabilistic Borel measure in $\mathbb{R}^{2}$ supported on graph $\left.f\right|_{\mathcal{I}}$ defined by

$$
\nu=\left(\operatorname{id}_{\mathbb{R}}, f\right)_{*} \mu
$$

By definition, we have

$$
\nu(U)=\mu(\{x \in \mathbb{R}:(x, f(x)) \in U\})
$$

for every Borel set $U \subset \mathbb{R}^{2}$.

Recall that by Proposition 18 (c), we assume that $d_{n} \rightarrow \infty$ as $n \rightarrow \infty$, in particular $d_{n}>1$ and $\log ^{+} d_{n}=\log d_{n}$.

For $t \in \mathcal{I}$ and a small $r>0$, let $Q_{r}(t)$ be the square with horizontal and vertical sides of length $r$ centred at $(t, f(t)) \in \operatorname{graph} f$.

Proposition 22. For every $t \in \mathcal{I}$, we have

$$
\liminf _{r \rightarrow 0^{+}} \frac{\log \nu\left(Q_{r}(t)\right)}{\log r} \geq 1+\liminf _{n \rightarrow \infty} \frac{\log d_{n}}{\log \left(b_{n} d_{n} / d_{n+1}\right)} .
$$

Before proving Proposition 22, we state two lemmas. As previously, take $k=k(r), m=$ $m(r) \in \mathbb{N}$ such that (11) is satisfied. Let $l=l(r) \in \mathbb{N}$ be such that

$$
a_{l+1} \leq r<a_{l} .
$$

Lemma 23. We have

$$
\lim _{r \rightarrow 0^{+}} \frac{l(r)}{-\log r}=0 .
$$

Proof. If $l \leq k$, then by (44) and (11), we have $l /(-\log r)=o(1)$ as $r \rightarrow 0^{+}$. Suppose now $l>k$. Let

$$
A_{n}=\inf _{i \geq n} \frac{a_{i}}{a_{i+1}} .
$$


Since $a_{n+1} / a_{n} \rightarrow 0$, we have $A_{n+1} \geq A_{n}$ and $A_{n} \rightarrow \infty$. By definition,

$$
r<a_{l}=\frac{a_{l}}{a_{l-1}} \cdots \frac{a_{k+1}}{a_{k}} a_{k} \leq A_{k}^{-(l-k)} a_{k}<A_{k}^{-(l-k)} .
$$

This together with (44) implies

$$
0<\frac{l}{-\log r}=\frac{l-k}{-\log r}+\frac{k}{-\log r}<\frac{1}{\log A_{k}}+\frac{k}{\log b_{k}} \rightarrow 0
$$

as $r \rightarrow 0^{+}$.

Let

$$
\mathcal{M}_{n}=\left\{j \in \mathcal{J}_{n}:\left.\operatorname{graph} f\right|_{I_{n, j}} \cap Q_{r}(t) \neq \emptyset\right\}
$$

for $n \in \mathbb{N}$. Since the intervals of $n$-th generation are separated by gaps of length at least $(1-|I|) / b_{n}$, by (11) we have

$$
\operatorname{card} \mathcal{M}_{k} \leq 2, \quad \operatorname{card} \mathcal{M}_{k+1} \leq m+2 .
$$

Lemma 24. There exists a constant $c_{3}>0$ such that for every $t \in \mathcal{I}, r>0$ and $n \in \mathbb{N}$, we have $\operatorname{card} \mathcal{M}_{n} \neq 0$ and

$$
\frac{\operatorname{card} \mathcal{M}_{n+1}}{\operatorname{card} \mathcal{M}_{n}} \leq \begin{cases}c_{3} d_{n+1} / d_{n} & \text { if } n<l, \\ c_{3} & \text { if } n \geq l \text { and } d_{n} / b_{n+1}>r, \\ c_{3} r b_{n+1} / d_{n} & \text { if } n \geq l \text { and } d_{n} / b_{n+1} \leq r .\end{cases}
$$

Proof. Since $t \in \mathcal{I}$, we have card $\mathcal{M}_{n} \neq 0$ for every $n \in \mathbb{N}$. Moreover,

$$
\frac{\operatorname{card} \mathcal{M}_{n+1}}{\operatorname{card} \mathcal{M}_{n}} \leq \max _{j \in \mathcal{J}_{n}} \operatorname{card} \mathcal{M}_{n+1}(j),
$$

where

$$
\mathcal{M}_{n+1}(j)=\left\{j^{\prime} \in \mathcal{M}_{n+1}: I_{n+1, j^{\prime}} \subset I_{n, j}\right\} .
$$

Take $j \in \mathcal{J}_{n}$ and let

$$
\begin{aligned}
& x=\max \left\{u \in I_{n, j}:(u, f(u)) \in Q_{r}(t)\right\}, \\
& y=\min \left\{u \in I_{n, j}:(u, f(u)) \in Q_{r}(t)\right\} .
\end{aligned}
$$

By definition, the interval $[y, x]$ intersects all the intervals $I_{n+1, j^{\prime}} \subset I_{n, j}$ with $j^{\prime} \in \mathcal{M}_{n+1}$. Since the intervals of $(n+1)$-th generation have length $|I| / b_{n+1}$ and are separated by gaps of length at least $(1-|I|) / b_{n+1}$, this implies

$$
\text { card } \mathcal{M}_{n+1}(j) \leq(x-y) b_{n+1}+1+|I| .
$$

By Lemma 17,

$$
r \geq|f(x)-f(y)| \geq c_{1} d_{n}(x-y)-c_{2} a_{n+1},
$$

which implies

$$
x-y \leq \frac{r+c_{2} a_{n+1}}{c_{1} d_{n}}<c \frac{r+a_{n+1}}{d_{n}}
$$


for a suitable constant $c>0$, so (22) gives

$$
\operatorname{card} \mathcal{M}_{n+1}(j) \leq c \frac{\left(r+a_{n+1}\right) b_{n+1}}{d_{n}}+1+|I| \text {. }
$$

Suppose $n<l$ for $l$ from (19). Then $a_{n+1}>r$, so (23) gives

$$
\text { card } \mathcal{M}_{n+1}(j)<2 c \frac{a_{n+1} b_{n+1}}{d_{n}}+1+|I| \text {. }
$$

Let $c_{3}=2 c+1+|I|$. If $a_{n+1} b_{n+1}<d_{n}$, then by (24),

$$
\operatorname{card} \mathcal{M}_{n+1}(j) \leq c_{3}<c_{3} \frac{d_{n+1}}{d_{n}} \text {. }
$$

Otherwise, if $a_{n+1} b_{n+1} \geq d_{n}$, then by (24),

$$
\operatorname{card} \mathcal{M}_{n+1}(j) \leq c_{3} \frac{a_{n+1} b_{n+1}}{d_{n}}<c_{3} \frac{d_{n+1}}{d_{n}} .
$$

Suppose now $n \geq l$. Then $a_{n+1} \leq r$, so (23) gives

$$
\operatorname{card} \mathcal{M}_{n+1}(j) \leq 2 c \frac{r b_{n+1}}{d_{n}}+1+|I|
$$

If $d_{n} / b_{n+1}>r$, then by (27),

$$
\operatorname{card} \mathcal{M}_{n+1}(j)<c_{3} \text {. }
$$

Otherwise, if $d_{n} / b_{n+1} \leq r$, then by (27),

$$
\operatorname{card} \mathcal{M}_{n+1}(j) \leq c_{3} \frac{r b_{n+1}}{d_{n}}
$$

Using (21), (25), (26), (28) and (29), we end the proof of the lemma.

Proof of Proposition 22. Consider $k=k(r), m=m(r), l=l(r)$ from (11) and (19)). In view of Lemma 21, it is sufficient to show

$$
\frac{\log \nu\left(Q_{r}(t)\right)}{\log r} \geq 1+\max \left(\frac{\log d_{k}}{\log \left(b_{k+1} / m\right)}-o(1), \frac{\log \left(d_{k+1} / m\right)}{\log \left(b_{k+1} / m\right)}-o(1)\right)
$$

as $r \rightarrow 0^{+}$. First we show that

$$
\frac{\log \nu\left(Q_{r}(t)\right)}{\log r} \geq 1+\frac{\log d_{k}}{\log \left(b_{k+1} / m\right)}-o(1)
$$

as $r \rightarrow 0^{+}$. Let

$$
l_{1}=\max \{k, l\}, \quad s_{1}=\min \left\{n \geq l_{1}: d_{n} / b_{n+1} \leq r\right\} .
$$

By Lemma 14, the number $s_{1}$ is well-defined. By (20) and Lemma 24, we have

and

$$
0<\operatorname{card} \mathcal{M}_{s_{1}} \leq 2 c_{3}^{s_{1}-k} \frac{d_{l_{1}}}{d_{k}}
$$

$$
0<\operatorname{card} \mathcal{M}_{s_{1}+1} \leq 2 c_{3}^{s_{1}-k+1} \frac{d_{l_{1}} r b_{s_{1}+1}}{d_{k} d_{s_{1}}} \leq 2 c_{3}^{s_{1}-k+1} \frac{r b_{s_{1}+1}}{d_{k}}
$$


so by (18) and Lemma 14,

$$
0<\nu\left(Q_{r}(t)\right) \leq \mu\left(\bigcup_{j \in \mathcal{M}_{s_{1}}} I_{s_{1}, j}\right)<\frac{\operatorname{card} \mathcal{M}_{s_{1}}}{q^{s_{1}} b_{s_{1}}} \leq \frac{2 c_{3}^{s_{1}-k}}{q^{s_{1}}} \frac{d_{l_{1}}}{d_{k} b_{s_{1}}}<C^{s_{1}} \frac{b_{l_{1}}}{b_{s_{1}}} \leq \frac{C^{s_{1}}}{B_{l_{1}}^{s_{1}-l_{1}}}
$$

and

$$
0<\nu\left(Q_{r}(t)\right) \leq \mu\left(\bigcup_{j \in \mathcal{M}_{s_{1}+1}} I_{s_{1}+1, j}\right)<\frac{\operatorname{card} \mathcal{M}_{s_{1}+1}}{q^{s_{1}+1} b_{s_{1}+1}} \leq \frac{2 c_{3}^{s_{1}-k+1}}{q^{s_{1}+1}} \frac{r}{d_{k}}<C^{s_{1}} \frac{r}{d_{k}}
$$

where

$$
B_{n}=\inf _{i \geq n} \frac{b_{i+1}}{b_{i}}
$$

and $C>0$ is a suitable constant. (Since $b_{n+1} / b_{n} \rightarrow \infty$, we have $B_{n+1} \geq B_{n}$ and $B_{n} \rightarrow \infty$.) If

$$
\frac{C^{s_{1}}}{B_{l_{1}}^{s_{1}-l_{1}}} \leq r^{2}
$$

then by (32), Lemma 14 and (11),

$$
\frac{\log \nu\left(Q_{r}(t)\right)}{\log r} \geq 2>1+\frac{\log d_{k}}{\log b_{k}}>1+\frac{\log d_{k}}{\log \left(b_{k+1} / m\right)},
$$

so (31) is satisfied. Hence, we can assume

$$
\frac{C^{s_{1}}}{B_{l_{1}}^{s_{1}-l_{1}}}>r^{2}
$$

which implies

$$
\left(s_{1}-l_{1}\right) \log B_{l_{1}}-s_{1} \log C<-2 \log r .
$$

By (11) and (33),

$$
\frac{\log \nu\left(Q_{r}(t)\right)}{\log r} \geq 1+\frac{\log d_{k}}{\log \left(b_{k+1} / m\right)}-\frac{s_{1} \log C}{-\log r} .
$$

Now, if $s_{1} \leq 2 l_{1}$, then by (44) and Lemma 23, we have (31). Otherwise, if $s_{1}>2 l_{1}$, then by (34),

$$
\frac{s_{1} \log C}{-\log r}<\frac{4 \log C}{\log B_{l_{1}}-2 \log C} \rightarrow 0
$$

as $r \rightarrow 0^{+}$, so (31) holds.

Now we show that

$$
\frac{\log \nu\left(Q_{r}(t)\right)}{\log r} \geq 1+\frac{\log \left(d_{k+1} / m\right)}{\log \left(b_{k+1} / m\right)}-o(1)
$$

as $r \rightarrow 0^{+}$. The proof is analogous to the proof of (31). Let

$$
l_{2}=\max \{k+1, l\}, \quad s_{2}=\min \left\{n \geq l_{2}: d_{n} / b_{n+1} \leq r\right\} .
$$


By Lemma 14, the number $s_{2}$ is well-defined. By (20) and Lemma 24, we have

and

$$
\operatorname{card} \mathcal{M}_{s_{2}} \leq c_{3}^{s_{2}-k-1}(m+2) \frac{d_{l_{2}}}{d_{k+1}}
$$

$$
\operatorname{card} \mathcal{M}_{s_{2}+1} \leq c_{3}^{s_{2}-k}(m+2) \frac{d_{l_{2}} r b_{s_{2}+1}}{d_{k+1} d_{s_{2}}} \leq c_{3}^{s_{2}-k}(m+2) \frac{r b_{s_{2}+1}}{d_{k+1}}
$$

so by (18) and Lemma 14,

$$
\nu\left(Q_{r}(t)\right) \leq \mu\left(\bigcup_{j \in \mathcal{M}_{s_{2}}} I_{s_{2}, j}\right)<\frac{\operatorname{card} \mathcal{M}_{s_{2}}}{q^{s_{2}} b_{s_{2}}} \leq \frac{c_{3}^{s_{2}-k-1}(m+2)}{q^{s_{2}}} \frac{d_{l_{2}}}{d_{k+1} b_{s_{2}}}<C^{s_{2}} \frac{m b_{l_{2}}}{b_{s_{2}}} \leq C^{s_{2}} \frac{m}{B_{l_{2}}^{s_{2}-l_{2}}}
$$

and

$$
\nu\left(Q_{r}(t)\right) \leq \mu\left(\bigcup_{j \in \mathcal{M}_{s_{2}+1}} I_{s_{2}+1, j}\right)<\frac{\operatorname{card} \mathcal{M}_{s_{2}+1}}{q^{s_{2}+1} b_{s_{2}+1}} \leq \frac{c_{3}^{s_{2}-k}(m+2)}{q^{s_{2}+1}} \frac{r}{d_{k+1}}<C^{s_{2}} \frac{m r}{d_{k+1}}
$$

for a suitable constant $C>0$. If

then by (36), Lemma 14 and (11),

$$
\frac{C^{s_{2}}}{B_{l_{2}}^{s_{2}-l_{2}}} \leq r^{2}
$$

$$
\frac{\log \nu\left(Q_{r}(t)\right)}{\log r} \geq 2>1+\frac{\log \left(d_{k+1} / m\right)}{\log \left(b_{k+1} / m\right)},
$$

so (35) holds. Hence, we can assume

which implies

$$
\frac{C^{s_{2}}}{B_{l_{2}}^{s_{2}-l_{2}}}>r^{2}
$$

$$
\left(s_{2}-l_{2}\right) \log B_{l_{2}}-s_{2} \log C<-2 \log r .
$$

By (11) and (37),

$$
\frac{\log \nu\left(Q_{r}(t)\right)}{\log r} \geq 1+\frac{\log \left(d_{k+1} / m\right)}{\log \left(b_{k+1} / m\right)}-\frac{s_{2} \log C}{-\log r} .
$$

If $s_{2} \leq 2 l_{2}$, then by (44) and Lemma 23, we have (35). Otherwise, if $s_{2}>2 l_{2}$, then by (38),

$$
\frac{s_{2} \log C}{-\log r}<\frac{4 \log C}{\log B_{l_{2}}-2 \log C} \rightarrow 0
$$

as $r \rightarrow 0^{+}$, so (35) is satisfied.

In this way we showed (31) and (35), which implies (30) and ends the proof of the proposition.

Proposition 22 together with Proposition 18 (c) and Lemma 13 give immediately the following corollary. 


\section{Corollary 25.}

$$
\operatorname{dim}_{H}\left(\left.\operatorname{graph} f\right|_{\mathcal{I}}\right) \geq 1+\liminf _{n \rightarrow \infty} \frac{\log ^{+} d_{n}}{\log \left(b_{n+1} d_{n} / d_{n+1}\right)}
$$

Propositions $19 \sqrt[20]{20}$ and Corollary 25 end the proof of Theorem 1.

\section{REFERENCES}

[1] A. S. Besicovitch and H. D. Ursell, Sets of fractional dimensions (V): On dimensional numbers of some continuous curves, J. London Math. Soc. 12 (1937), 18-25.

[2] A. Carvalho, Hausdorff dimension of scale-sparse Weierstrass-type functions, Fund. Math. 213 (2011), $1-13$.

[3] K. J. Falconer, Fractal Geometry: Mathematical Foundations and Applications, J. Wiley \& Sons, 1990.

[4] Y. Heurteaux, Weierstrass functions with random phases, Trans. Amer. Math. Soc. 335 (2003), 30653077.

[5] B. R. Hunt, Hausdorff dimension of graphs of Weierstrass functions, Proc. Amer. Math. Soc. 126 (1998), 791-800.

[6] F. Ledrappier, On the dimension of some graphs, Contemp. Math. 135 (1992), 285-293.

[7] Y.-Y. Liu, A function whose graph is of dimension 1 and has locally an infinite one-dimensional Hausdorff measure, C. R. Acad. Sci. Paris Sr. I Math. 332 (2001), 19-23.

[8] P. Mattila, Geometry of sets and measures in Euclidean spaces, Cambridge Univ. Press, Cambridge, 1995.

[9] R. D. Mauldin and S. C. Williams, On the Hausdorff dimension of some graphs, Trans. Amer. Math. Soc. 298 (1986), 793-804.

[10] F. Przytycki and M. Urbański, On the Hausdorff dimension of some fractal sets, Studia Math. 93 (1989), 155-186.

[11] P. Wingren, Concerning a real-valued continuous function on the interval with graph of Hausdorff dimension 2, L'Enseign. Math. 41 (1995) 103-110.

Institute of Mathematics, University of Warsaw, Ul. Banacha 2, 02-097 WarszaWA, POLAND

E-mail address: baranski@mimuw.edu.pl 